\title{
Secondary Chiari malformation due to enlarged spinal arachnoid villi-like structure: illustrative case
}

\author{
Izumi Koyanagi, MD, Yasuhiro Chiba, MD, Hiroyuki Imamura, MD, Masami Yoshino, MD, and Toshimitsu Aida, MD \\ Department of Neurosurgery, Hokkaido Neurosurgical Memorial Hospital, Sapporo, Hokkaido, Japan
}

\begin{abstract}
BACKGROUND Secondary Chiari malformation can be caused by various disorders associated with cerebrospinal fluid (CSF) leakage at the spinal level. In this report, the authors describe a rare case of secondary Chiari malformation caused by excessive CSF absorption through the enlarged spinal arachnoid villi-like structure.

OBSERVATIONS A20-year-old woman presented with progressive severe headache and posterior neck pain. Magnetic resonance imaging showed tonsillar herniation and decreased subarachnoid space around the spinal cord. A hypointense signal area was observed in the ventral spinal canal on a T2-weighted image. An axial image revealed multiple small, arachnoid cyst-like structures at the right $\mathrm{T} 1$ nerve root sleeve. Direct surgery revealed that the cyst-like structures were continuous with the arachnoid membrane and protruded into the abnormally large epidural venous sinus. The cyst-like structures were resected, and the dural sleeve was repaired using fascia. The patient showed good improvement of symptoms after surgery.

LESSONS Excessive CSF absorption through the enlarged spinal arachnoid villi-like structure can cause secondary Chiari malformation. Neurosurgeons should be aware of this unusual mechanism of CSF leakage. Simple posterior fossa decompression will be ineffective or even harmful.
\end{abstract}

https://thejns.org/doi/abs/10.3171/CASE2049

KEYWORDS CSF leakage; secondary Chiari malformation; spinal arachnoid villi; spinal venous sinus

Cerebrospinal fluid (CSF) leakage at the spinal level can cause descent of the cerebellar tonsil and/or the displaced brainstem (secondary Chiari malformation) with intracranial hypotension syndrome. Osteolytic disorders such as Gorham-Stout disease, ${ }^{1,2}$ dural ectasia,${ }^{3}$ extradural arachnoid diverticula,${ }^{4,5}$ trauma, ${ }^{6}$ or spinal surgery ${ }^{7}$ have been reported as the etiologies of CSF leakage. In this report, we present a rare case of secondary Chiari malformation caused by excessive absorption of CSF from the enlarged spinal arachnoid villi-like structure at the T1 nerve root sleeve into the spinal venous sinus.

\section{Illustrative Case}

A 20-year-old woman presented with progressive aggravation of posterior headache and neck pain of 3 months' duration before admission. She had been seen in follow-up at another outpatient clinic under the diagnosis of migraine and Chiari type 1 malformation since the age of 15 years. Because of aggravated tonsillar herniation and headache, she was referred to our hospital. On admission, she complained of severe posterior headache and neck pain. Except for severe pain, the patient showed no cranial nerve or spinal cord symptoms. Cervical magnetic resonance imaging (MRI) showed tonsillar descent to the level of the C2 lamina and a tight foramen magnum (Fig. $1 A$ and $B$ ). An enlarged central canal was present at C6-T1. A hypointense signal area was observed ventral to the anterior subarachnoid space on a T2-weighted image (Fig. 1B). An axial T2-weighted image showed that the right intervertebral foramen was enlarged at $\mathrm{T} 1 / 2$ and that the hypointense signal area extended to the intervertebral foramina at this level (Fig. 1C). A heavy T2-weighted axial image (fast imaging employing steadystate acquisition) revealed that multiple arachnoid cyst-like structures were present at the right T1 nerve root sleeve adjacent to the hypointense area (Fig. 1D). The subarachnoid space around the spinal cord was narrowed at this level. Computed tomography (CT) showed bone erosion at the right pedicle and lamina at the T1 vertebra (Fig. 2). Thoracic MRI revealed an enlarged dorsal epidural fat space and decreased subarachnoid space at the thoracic levels (Fig. 3). Brain CT displayed narrowed intracranial ventricles and subarachnoid cisterns around the brainstem. On the basis of these

ABBREVIATIONS CSF = cerebrospinal fluid; $\mathrm{CT}=$ computed tomography; FIESTA = fast imaging employing steady-state acquisition; $\mathrm{MRI}=$ magnetic resonance imaging INCLUDE WHEN CITING Published January 11, 2021; DOI: 10.3171/CASE2049.

SUBMITTED October 3, 2020. ACCEPTED October 15, 2020.

(c) 2021 The authors, CC BY-NC-ND 4.0 (http://creativecommons.org/licenses/by-nc-nd/4.0/) 

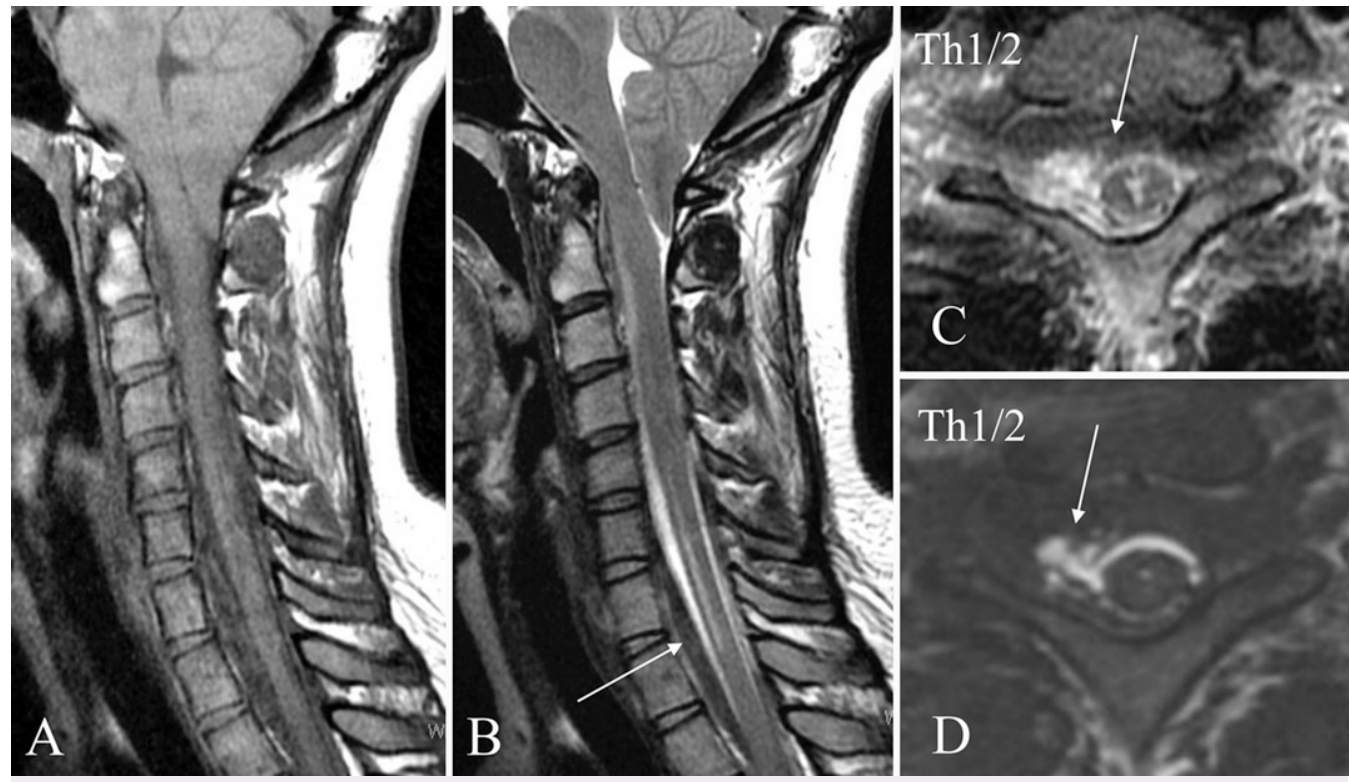

FIG. 1. Preoperative MRI findings of the cervical spine. A: T1-weighted sagittal image. B: T2-weighted sagittal image. C: T2-weighted axial image at T1/2. D: Heavy T2-weighted axial image (fast imaging employing steady-state acquisition [FIESTA]) at T1/2. The T1- and T2-weighted images demonstrate descent of the cerebellar tonsil and a tight foramen magnum. T2-weighted sagittal image shows hypointense signal area (arrow, B) at the ventral side of the spinal canal below $\mathrm{C} 6$ and an enlarged central canal at C5-T1. T2-weighted axial image shows the hypointense area (arrow, C) extending to the intervertebral foramen. FIESTA image reveals multiple small, arachnoid cyst-like structures (arrow, D) at the right T1 nerve root sleeve. Subarachnoid space around the spinal cord is narrowed.

imaging findings and clinical symptoms, CSF leakage from the arachnoid cyst-like structures at the right $\mathrm{T} 1$ nerve root sleeve was considered to have caused secondary Chiari malformation and intracranial hypotension syndrome.

The patient underwent right upper thoracic hemilaminectomy in a prone position under general anesthesia. At the first surgery, we encountered massive hemorrhage from the epidural space after hemilaminectomy and found no arachnoid cyst-like structures. However, postoperative CT revealed that the hemilaminectomy was made mainly at the T2-T3 levels, so a second surgery was performed the next day. Right hemilaminectomy was made at the $\mathrm{T} 1$ and the lower half of the $\mathrm{C} 7$ levels. After removal of the ligamentum flavum, massive venous hemorrhage was again encountered. Hemorrhage was controlled using a gelatin-based hemostatic agent, cotton, and elevation of
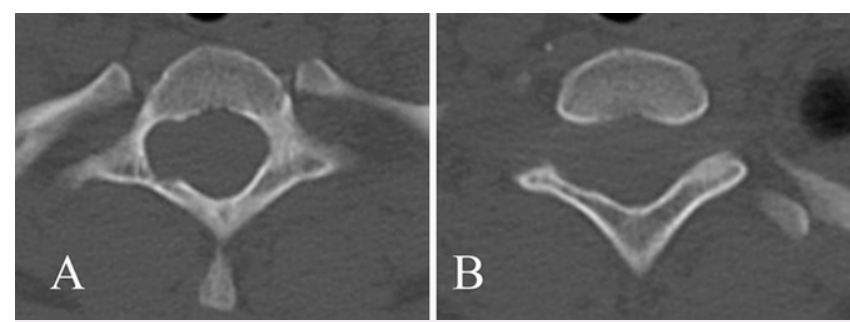

FIG. 2. Preoperative bone window CT findings of the upper thoracic spine. Axial images show bone erosion at the right pedicle and lamina of the T1 vertebra $(\mathbf{A})$ and an enlarged right intervertebral foramen at $\mathrm{T} 1 / 2(\mathrm{~B})$.
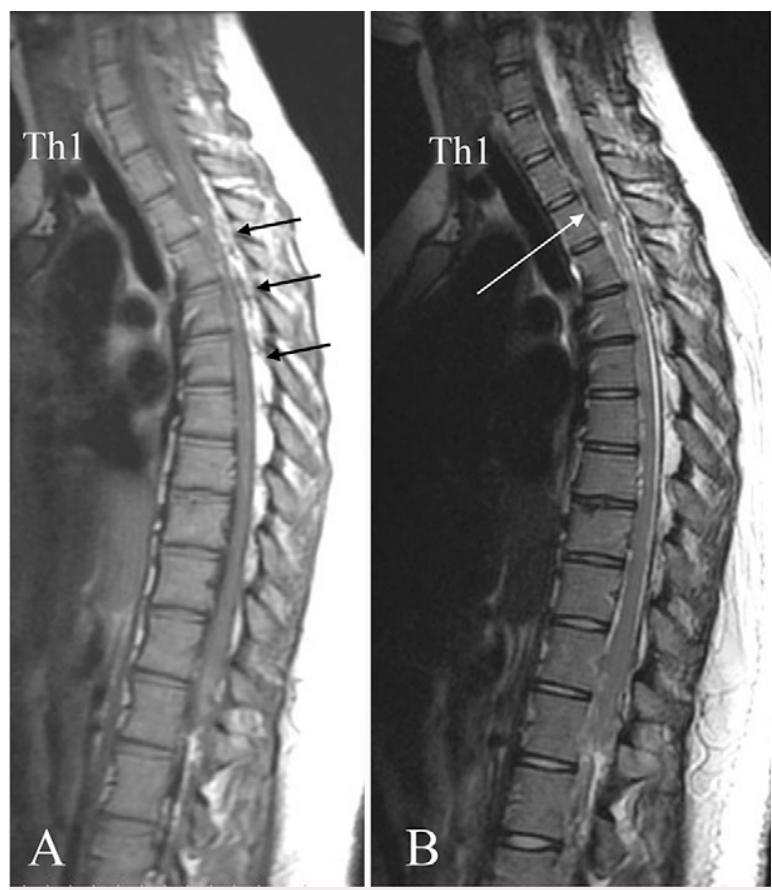

FIG. 3. Preoperative thoracic MRI findings. A: T1-weighted sagittal image shows increased thickness of the epidural fat tissue (black arrows). B: T2-weighted sagittal image shows decreased subarachnoid space around the spinal cord. The hypointense signal area at the ventral side of the spinal canal extends to the T3 vertebral body level (white arrow). 


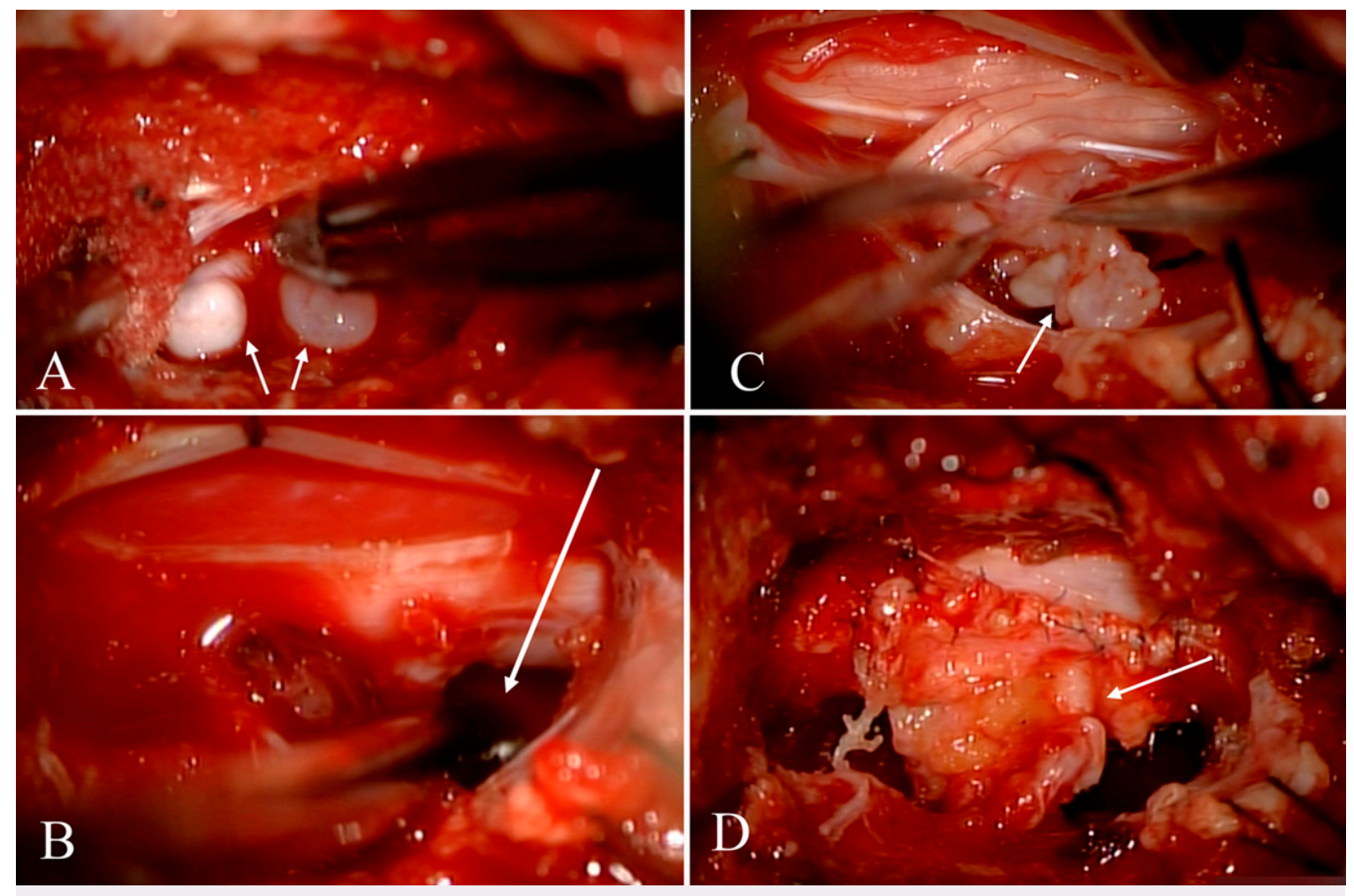

FIG. 4. Intraoperative findings in the second operation. The right $\mathrm{T} 1$ lamina and the lower part of the $\mathrm{C} 7$ lamina were removed by drilling. Rostral is to the right, and midline is to the top. A: Multiple small, arachnoid cyst-like structures (arrows) are present at the right T1 nerve root sleeve. B: The spinal dural tube is surrounded by the large venous sinus. Arrow indicates the ventral side of the venous sinus. The dura is opened at the midline of the hemilaminectomy window. C: After opening the dura in a T-shaped fashion to the right $\mathrm{T} 1$ nerve root sleeve. The multiple small, arachnoid cyst-like structures (arrow) are continuous with arachnoid membrane of the right T1 nerve root. D: After repair of the dural opening site using autologous femoral fascia (arrow).
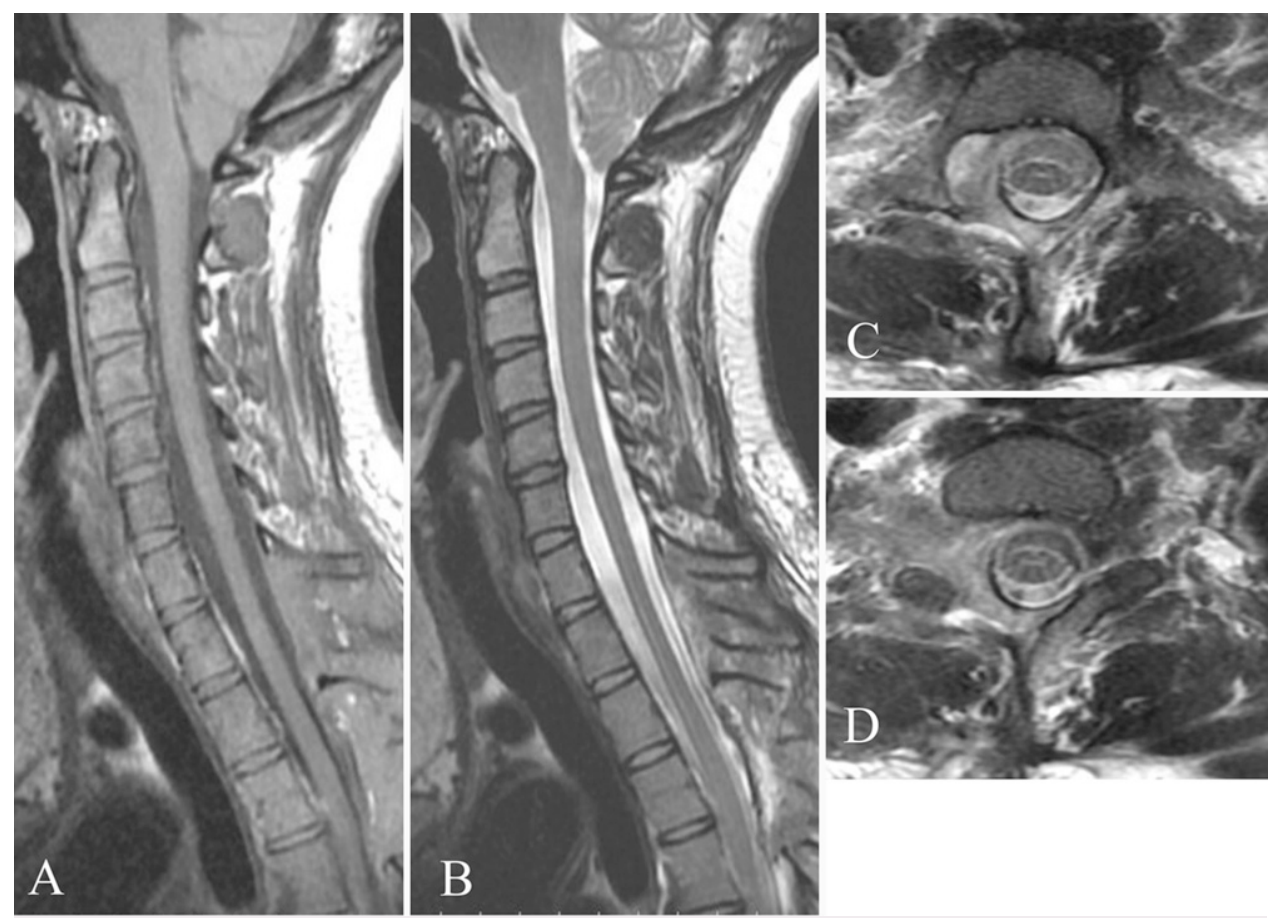

FIG. 5. Postoperative MRI findings of the cervical spine 1 month after the surgery. A: T1-weighted sagittal image. B: T2-weighted sagittal image. C, D: T2-weighted axial images at the T1 (C) and T1/2 (D) levels. Sagittal images show elevated cerebellar tonsil to the $\mathrm{C} 1$ level and enlarged subarachnoid space at the foramen magnum. The hypointense signal area is not present on the T2-weighted sagittal image. T2weighted axial images at $\mathrm{T} 1$ and $\mathrm{T} 1 / 2$ demonstrate enlarged subarachnoid space around the spinal cord. The hypointense signal area has disappeared. 
the upper body of the patient. This time, we found multiple small, arachnoid cyst-like structures at the right $\mathrm{T} 1$ nerve root sleeve (Fig. 4A). A venous sinus-like structure was observed around the dural tube, especially at the ventral side (Fig. 4B). The arachnoid cyst-like structures protruded into this large epidural venous sinus from the T1 nerve root sleeve. The dura was opened in a T-shaped fashion to the right T1 root sleeve. The arachnoid cyst-like structures were continuous with the arachnoid membrane at the right $\mathrm{T} 1$ nerve root(Fig. 4C). This structure was resected, and the dura was repaired using femoral fascia (Fig. 4D). After surgery, the patient's severe headache and posterior neck pain resolved. Histologically, the arachnoid cyst-like structures showed arachnoid cells with proliferation of collagen fibers.

Postoperative MRI showed improved tonsillar herniation and disappearance of the intraspinal canal hypointense area ventral to the spinal cord (Fig. 5). The subarachnoid space around the spinal cord also recovered. Brain MRI showed normal-sized intracranial ventricles and subarachnoid cisterns around the brainstem. The patient developed double vision most likely due to incomplete abducens palsy 4 weeks after surgery. These symptoms disappeared spontaneously approximately 2 weeks after onset. The patient was well for 8 months after surgery.

\section{Discussion \\ Observations}

On the basis of the preoperative imaging and intraoperative findings, tonsillar and brainstem descent was caused by CSF leakage at the arachnoid cyst-like structures of the right T1 nerve root sleeve. The hypointense area ventral to the spinal cord at the cervicothoracic levels on a preoperative T2-weighted MRI scan was the large venous sinus around the spinal dural tube. It is likely that the arachnoid cyst-like structures in this patient were the enlarged arachnoid villi at the spinal nerve root. Arachnoid villi are the protrusions of arachnoid membrane into the dural sinus. They are usually present at the intracranial venous sinuses, most prominent at the superior sagittal sinus, and are supposed to be the main route of CSF absorption into the venous system. It has been known that the arachnoid villi are also present at the spinal root levels. ${ }^{8,9}$ Using cadaver specimens, Kido et al. ${ }^{8}$ reported that the human spinal arachnoid villi were distributed on almost every nerve root in the thoracic and lumbar regions. They were small in size and closely related to the venous sinuses. According to the cadaver study by Tubbs et al., ${ }^{9}$ the human spinal arachnoid villi were more concentrated in the lumbar region and intimately related to the adjacent radicular vein. The size of the spinal arachnoid villi was reported to be $50-170 \mu \mathrm{m} .{ }^{9}$ The spinal arachnoid villi were also supposed to be the pathway of CSF absorption into the venous system. ${ }^{8,9}$ In the present patient, it is likely that excessive CSF absorption occurred through the enlarged arachnoid villi-like structure into the spinal venous sinus and caused secondary Chiari malformation. It is still unknown whether the abnormally large spinal venous sinus was the result of receiving excessive CSF or was congenital in origin. CT findings of expansive bone changes at the intervertebral foramen suggested that the enlarged arachnoid villi-like structure and surrounding epidural venous plexus or sinus had been present for a long time. The venous plexus or sinus around the T1 nerve root sleeve presumably extended rostrocaudally with age and gained more CSF absorption ability related to negative pressure of the thoracic cavity. Recently, a CSF venous fistula was reported as the cause of spinal CSF leakage. According to the article by Farb et al., ${ }^{10} \mathrm{CSF}$ was shown to leak into the adjacent venous channel through the pseudomeningocele at the spinal root level. However, in our case, the arachnoid villi-like structure was entirely located in the large venous sinus. This pathomechanism of secondary Chiari malformation has never been reported, to our knowledge.

\section{Lessons}

Excessive CSF absorption through the enlarged spinal arachnoid villi-like structure can cause secondary Chiari malformation. Neurosurgeons should be aware of this unusual mechanism of CSF leakage. Simple posterior fossa decompression will be ineffective or even harmful.

\section{References}

1. Stephens S, Squires L, Campbell R, et al. Multifocal GorhamStout disease associated with Chiari I malformation and recurrent aseptic meningitis: case report and review of literature. $\mathrm{J}$ Clin Neurosci. 2020;72:486-492.

2. Yoshimoto S, Takai K, Takahashi K, et al. Intracranial hypotension and hypertension: reversible Chiari malformation due to dynamic cerebrospinal fluid abnormalities in Gorham-Stout disease. Case report. J Neurosurg Pediatr. 2018;22(5):508-512.

3. Puget $\mathrm{S}$, Kondageski $\mathrm{C}$, Wray $\mathrm{A}$, et al. Chiari-like tonsillar herniation associated with intracranial hypotension in Marfan syndrome. Case report. J Neurosurg. 2007;106(1 Suppl):48-52.

4. Atkinson JL, Weinshenker BG, Miller GM, et al. Acquired Chiari I malformation secondary to spontaneous spinal cerebrospinal fluid leakage and chronic intracranial hypotension syndrome in seven cases. J Neurosurg. 1998;88(2):237-242.

5. Ohtonari T, Nishihara N, Ota T, et al. Rapid reduction of syrinx associated with traumatic intracranial hypotension by direct surgery: case report. Neurol Med Chir (Tokyo). 2009;49(2):66-70.

6. Hatae R, Kohri R, Maeda K, et al. A rare case of Chiari type-1 malformation accompanied by symptomatic cerebrospinal fluid hypovolemia: comparison of congenital Chiari type-1 malformation and acquired Chiari malformation secondary to cerebrospinal fluid hypovolemia: case report. Neurol Med Chir (Tokyo). 2014;54(7):558-562.

7. Samii $C$, Möbius $E$, Weber W, et al. Pseudo Chiari type I malformation secondary to cerebrospinal fluid leakage. J Neurol. 1999;246(3):162-164.

8. Kido DK, Gomez DG, Pavese AM Jr, et al. Human spinal arachnoid villi and granulations. Neuroradiology. 1976;11(5):221-228.

9. Tubbs RS, Hansasuta A, Stetler W, et al. Human spinal arachnoid villi revisited: immunohistological study and review of the literature. J Neurosurg Spine. 2007;7(3):328-331.

10. Farb RI, Nicholson PJ, Peng PW, et al. Spontaneous intracranial hypotension: a systematic imaging approach for CSF leak localization and management based on MRI and digital subtraction myelography. AJNR Am J Neuroradiol. 2019;40(4):745-753.

\section{Disclosures}

The authors report no conflict of interest concerning the materials or methods used in this study or the findings specified in this paper.

\section{Author Contributions}

Conception and design: Koyanagi, Yoshino, Aida. Acquisition of data: Koyanagi, Yoshino. Analysis and interpretation of data: Koyanagi, Aida Drafting the article: Koyanagi, Yoshino, Aida. Critically revising the article: Yoshino, Aida. Reviewed submitted version of manuscript: Koyanagi, Yoshino, Aida. Approved the final version of the manuscript on behalf of all authors: Koyanagi. Administrative/technical/material support: Chiba, Imamura. Study supervision: Chiba, Yoshino, Aida.

\section{Correspondence}

Izumi Koyanagi: Hokkaido Neurosurgical Memorial Hospital, Nishuku, Sapporo, Japan. koyanagi@hnsmhp.or.jp. 